\title{
SYMMETRIC PERIODIC ORBITS FOR THE COLLINEAR CHARGED 3-BODY PROBLEM
}

\author{
JAUME LLIBRE ${ }^{1}$ AND DURVAL J. TONON ${ }^{2}$
}

\begin{abstract}
In this paper we study the existence of periodic symmetric orbits of the 3-body problem when each body possess mass and an electric charge. The main technique applied in this study is the continuation method of Poincaré.
\end{abstract}

\section{INTRODUCTION}

One of the most relevant objects to study in the theory of dynamical systems is the $n$-body problem and many work has been done for understanding its dynamics. Thus the study of its periodic orbits is one of the main objectives. In this paper we focus the attention on the periodic orbits of the 3-body problem when the three bodies are collinear and charged.

Recently many distinct techniques and methods have been used to prove the existence of periodic orbits for the $n$-body problem, for example, averaging theory, numerical analysis, Melnikov functions, normal forms, variational methods, among others. One of the first analytical studies of the existence of periodic orbits for the $n$-body problem was done by Poincare in [13], and we apply his method to study the symmetric periodic orbits of the charged collinear 3-body problem.

There exists a large literature studying the existence of periodic solutions of the $n$-body problem, see [10] and [11] for example. More precisely, if we restrict our attention to the 3-body problem, Hénon in [8] has studied numerically the existence and stability of a class of symmetric rectilinear periodic orbits of the general problem of three bodies. In [2] the authors studied, numerically, families of symmetric periodic orbits for the collinear 3-body problem when the two non-central masses are equal. In [9] the singularity generated by the triple collision of bodies of the collinear 3-body problem is studied.

In [5] the authors studied the symmetric periodic orbits by the continuation method of Poincare of the collinear 3-body problem when the bodies

2010 Mathematics Subject Classification. Primary 34C14, 34C20, 34C25.

Key words and phrases. symmetric periodic orbits, three body problem, Poincaré continuation method. 
do not have electric charges. In the present paper we allow that the bodies posses electric charges.

The continuation method of Poincaré was originally presented in [13] and this method consists in given a periodic solution for the system with a parameter equal to zero and it provides conditions for extending this solution to small values of the parameter. For more details about this method see for example [6].

The organization of this paper is as follows. In Section 2 the equations that model the dynamic of the collinear charged 3-body problem are described, in Section 3 we present the main results, and in Section 4 we study the symmetries of the periodic solutions of this system. The study of symmetric periodic orbits for the parameter $\mu=0$ are done in Section 5, and in Section 6 we apply the continuation method of Poincaré to extend the periodic solution obtained in previous section for $\mu=0$ to small and positive values of the parameter $\mu$. A brief conclusion and some comments comparing the periodic orbits of the charged with the ones of the uncharged system are presented in Section 7.

\section{Equations of MOtion of the COLLinear ChaRged 3-BOdy PROBLEM}

The charged $n$-body problem studies the dynamics of $n$ particles with a positive mass and an electrostatic charge of any sign, moving under the influence of the respective Newtonian and Coulombian forces. There exist many studies for particular values of $n$, in [7] it is considered the charged rhomboidal four-body problem, in [1] the charged isosceles 3-body problem, in [4] the restricted charged four body problem and in [12] the central configurations of the charged 3-body problem, among others.

In this paper we study the charged collinear 3-body problem. Taking conveniently the units of mass and charges we can assume, without loss of generality, that the gravitational constant and the Coulomb's constant are equal to one. The particles posses masses $m_{1}, m_{2}$ and $m_{3}$ with charges $q_{1}, q_{2}$ and $q_{3}$ in the position $x_{1}, x_{2}, x_{3} \in \mathbb{R}$, respectively.

The differential equations that governs the motion are given by

$$
m_{i} \ddot{x}_{i}=\nabla_{x_{i}} U,
$$

for $i=1,2,3$ where

$$
U\left(x_{1}, x_{2}, x_{3}\right)=\frac{\lambda_{12}}{d\left(x_{1}, x_{2}\right)}+\frac{\lambda_{13}}{d\left(x_{1}, x_{3}\right)}+\frac{\lambda_{23}}{d\left(x_{2}, x_{3}\right)},
$$

the symbol $\nabla_{x_{i}} U$ denotes the gradient of $U$ with respect to $x_{i}, \lambda_{i j}=m_{i} m_{j}-$ $q_{i} q_{j}$ for $i, j=1,2,3$ and $i \neq j$, and $d\left(x_{i}, x_{j}\right)$ denotes the Euclidean distance between the points $x_{i}$ and $x_{j}$. 
We denote the masses and electric charges of the bodies $b_{1}, b_{2}$ and $b_{3}$ by $m_{1}=\mu(1-\nu), m_{2}=1-\mu, m_{3}=\mu \nu$ and $\lambda_{1}=\mu \alpha, \lambda_{2}=\mu^{2} \beta, \lambda_{3}=\mu \gamma$, respectively, where $0 \leq \mu<1,0<\nu<1$ and $\alpha, \beta, \gamma \in \mathbb{R}$. We assume that the three bodies are in position $x_{i}$ such that $0<x_{1}<x_{2}<x_{3}$, see Figure 1 .

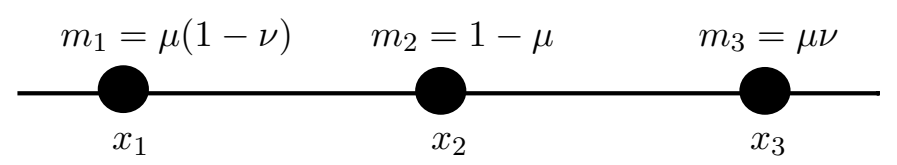

Figure 1. Position of the bodies in the collinear case.

Consider the change of coordinates given by $z_{1}=x_{2}-x_{1}$ and $z_{2}=x_{3}-x_{2}$, that denotes the distance between $x_{2}$ and $x_{1}$ and $x_{3}$ and $x_{2}$. Then the kinetic $T$ and the potential $U$ energies of the 3-body problem are given by

$$
\begin{aligned}
T\left(z_{1}, z_{2}\right)= & \frac{1}{2}\left(\mu(1-\nu)(1-\mu(1-\nu)) \dot{z}_{1}^{2}+2 \mu^{2} \nu(1-\nu) \dot{z}_{1} \dot{z}_{2}\right. \\
& \left.+\mu \nu(1-\mu \nu) \dot{z}_{2}^{2}+C^{2}\right), \\
U\left(z_{1}, z_{2}\right)= & -\left(\frac{\lambda_{12}}{z_{1}}+\frac{\lambda_{13}}{z_{1}+z_{2}}+\frac{\lambda_{23}}{z_{2}}\right),
\end{aligned}
$$

where $C=m_{1} \dot{q}_{1}+m_{2} \dot{q}_{2}+m_{3} \dot{q}_{3}$ is the linear momentum. With this notation we have that if $\lambda_{i j}>0$ then the resultant force between the particles $i$ and $j$ is attractive, and if $\lambda_{i j}<0$ then its repulsive. Without loss of generality we suppose that $C=0$, i.e. the center mass is in rest. Writing $L=T-U$ and denoting the new variables by

$$
p_{1}=\frac{\partial L}{\partial \dot{z}_{1}} \text { and } p_{2}=\frac{\partial L}{\partial \dot{z}_{2}}
$$

we obtain the Hamiltonian $H$ of the system given by

$$
\begin{aligned}
H= & \frac{p_{1}^{2}\left(-z_{1}\right) z_{2}(\mu \nu-1)\left(z_{1}+z_{2}\right)}{2(\mu-1) \mu(\nu-1) z_{1} z_{2}\left(z_{1}+z_{2}\right)} \\
& \frac{-2(\mu-1) \mu^{2}(\nu-1)\left(\left(z_{1}+z_{2}\right)\left(\gamma z_{1}+\alpha z_{2}\right)+\beta \mu z_{1} z_{2}\right)}{2(\mu-1) \mu(\nu-1) z_{1} z_{2}\left(z_{1}+z_{2}\right)} \\
& +\frac{p_{1} p_{2}}{\mu-1}-\frac{p_{2}^{2}(\mu(\nu-1)+1)}{2(\mu-1) \mu \nu} .
\end{aligned}
$$


Associated to the Hamiltonian (1) we have the system

$$
\begin{aligned}
\frac{d z_{1}}{d t} & =\frac{p_{1}}{\mu-\mu \nu}+\frac{p_{2}-p_{1}}{\mu-1}, \\
\frac{d z_{2}}{d t} & =\frac{p_{1}-p_{2}}{\mu-1}+\frac{p_{2}}{\mu \nu} \\
\frac{d p_{1}}{d t} & =\mu\left(-\frac{\alpha}{z_{1}^{2}}-\frac{\beta \mu}{\left(z_{1}+z_{2}\right)^{2}}\right), \\
\frac{d p_{2}}{d t} & =\mu\left(-\frac{\beta \mu}{\left(z_{1}+z_{2}\right)^{2}}-\frac{\gamma}{z_{2}^{2}}\right) .
\end{aligned}
$$

By the rescaling of the variables $z_{1}=\mu^{2} \widetilde{z}_{1}, z_{2}=\mu^{2} \widetilde{z}_{2}, t=\mu^{3} \widetilde{t}$ system (2) becomes

$$
\begin{aligned}
\frac{d z_{1}}{d t} & =\mu\left(\frac{p_{1}}{\mu-\mu \nu}+\frac{p_{2}-p_{1}}{\mu-1}\right), \\
\frac{d z_{2}}{d t} & =\frac{\mu\left(p_{1}-p_{2}\right)}{\mu-1}+\frac{p_{2}}{\nu}, \\
\frac{d p_{1}}{d t} & =-\frac{\alpha}{z_{1}^{2}}-\frac{\beta \mu}{\left(z_{1}+z_{2}\right)^{2}}, \\
\frac{d p_{2}}{d t} & =-\frac{\beta \mu}{\left(z_{1}+z_{2}\right)^{2}}-\frac{\gamma}{z_{2}^{2}}
\end{aligned}
$$

where we omit the tilde in the variables. The Hamiltonian associated to system (3) is given by

$$
H=\frac{1}{2}\left(-\frac{p_{1}^{2}}{\nu-1}-\frac{\mu\left(p_{1}-p_{2}\right)^{2}}{\mu-1}+\frac{p_{2}^{2}}{\nu}-\frac{2 \alpha}{z_{1}}-\frac{2 \beta \mu}{z_{1}+z_{2}}-\frac{2 \gamma}{z_{2}}\right) .
$$

Similarly to the case of the uncharged particles system (3) possess one singularity at $z_{1}=0$, that corresponds to a binary collision between $m_{1}$ and $m_{2}$, another at $z_{2}=0$ which corresponds to the collision between $m_{2}$ and $m_{3}$, and finally one at $z_{1}+z_{2}=0$ which represents a triple collision. Doing a Levi-Civita transformation, see [9], given by

$$
z_{1}=\xi_{1}^{2}, z_{2}=\xi_{2}^{2}, p_{1}=\frac{\eta_{1}}{2 \xi_{1}}, p_{2}=\frac{\eta_{2}}{\xi_{2}} \text { and } d t=4 \xi_{1}^{2} \xi_{2}^{2} d s
$$


the trajectories of the collinear charged 3-body problem (3) in the new coordinates are the solution of the system

$$
\begin{aligned}
\frac{d \xi_{1}}{d s}= & \frac{\xi_{2}\left(\eta_{1} \xi_{2}(1-\mu \nu)+\eta_{2} \mu(\nu-1) \xi_{1}\right)}{(\mu-1)(\nu-1)}, \\
\frac{d \xi_{2}}{d s}= & \frac{\xi_{1}\left(\eta_{1} \mu \nu \xi_{2}+\eta_{2} \xi_{1}(\mu(-\nu)+\mu-1)\right)}{(\mu-1) \nu}, \\
\frac{d \eta_{1}}{d s}= & \frac{-\eta_{1} \eta_{2} \mu \nu \xi_{2}\left(\xi_{1}^{2}+\xi_{2}^{2}\right)^{2}+\eta_{2}^{2} \xi_{1}(\mu(\nu-1)+1)\left(\xi_{1}^{2}+\xi_{2}^{2}\right)^{2}}{(\mu-1) \nu\left(\xi_{1}^{2}+\xi_{2}^{2}\right)^{2}} \\
& +\frac{8(\mu-1) \nu \xi_{1}\left(\beta \mu \xi_{2}^{4}+\gamma\left(\xi_{1}^{2}+\xi_{2}^{2}\right)^{2}+h \xi_{2}^{2}\left(\xi_{1}^{2}+\xi_{2}^{2}\right)^{2}\right)}{(\mu-1) \nu\left(\xi_{1}^{2}+\xi_{2}^{2}\right)^{2}}, \\
\frac{d \eta_{2}}{d s}= & \eta_{1}^{2} \xi_{2}\left(\frac{\mu(\nu-1)+\mu-1}{(\mu-1)(\nu-1)}\right)-\frac{\eta_{1} \eta_{2} \mu \xi_{1}}{\mu-1} \\
& +8 \xi_{2}\left(\alpha+\frac{\beta \mu \xi_{1}^{4}}{\left(\xi_{1}^{2}+\xi_{2}^{2}\right)^{2}}+h \xi_{1}^{2}\right)
\end{aligned}
$$

on the energy level $H=h$ for some constant $h$. System (4) is a Hamiltonian system with a Hamiltonian $G$ given by

$$
\begin{aligned}
G= & \frac{1}{2} \xi_{1}^{2} \xi_{2}^{2}\left(-\frac{8 \alpha}{\xi_{1}^{2}}-\frac{8 \beta \mu}{\xi_{1}^{2}+\xi_{2}^{2}}-\frac{8 \gamma}{\xi_{2}^{2}}-\frac{\eta_{1}^{2}}{(\nu-1) \xi_{1}^{2}}\right. \\
& \left.-\frac{\mu\left(\eta_{2} \xi_{1}-\eta_{1} \xi_{2}\right)^{2}}{(\mu-1) \xi_{1}^{2} \xi_{2}^{2}}+\frac{\eta_{2}^{2}}{\nu \xi_{2}^{2}}-8 h\right),
\end{aligned}
$$

with $G=0$ if and only if $H=h$. Note that system (4) is analytic except when $\xi_{1}^{2}+\xi_{2}^{2}=0$, that corresponds to the triple collision.

We study the periodic orbits of the collinear charged 3-body problem with binary collisions between $m_{1}, m_{2}$ and $m_{2}, m_{3}$. Considering these periodic solutions, our objective is study the periodic solutions of system (4) for $\mu>0$ sufficiently small, satisfying the energy relation $G=0$, more precisely we are interested in the symmetric periodic orbits of system (4). In this way will be necessary to study the symmetries involving this system.

\section{Statements of the main Results}

Consider the involutions

$$
\begin{aligned}
& S_{1}\left(\xi_{1}, \xi_{2}, \eta_{1}, \eta_{2}, s\right)=\left(-\xi_{1}, \xi_{2}, \eta_{1},-\eta_{2},-s\right), \\
& S_{2}\left(\xi_{1}, \xi_{2}, \eta_{1}, \eta_{2}, s\right)=\left(\xi_{1},-\xi_{2},-\eta_{1}, \eta_{2},-s\right), \\
& S_{3}\left(\xi_{1}, \xi_{2}, \eta_{1}, \eta_{2}, s\right)=\left(\xi_{1}, \xi_{2},-\eta_{1},-\eta_{2},-s\right) .
\end{aligned}
$$

A solution $\varphi(s)=\left(\xi_{1}(s), \xi_{2}(s), \eta_{1}(s), \eta_{2}(s)\right)$ of the differential equation is invariant under the symmetry $S_{i}$ if $S_{i}(\varphi(s))$ is also a solution of differential 
equation for $i=1,2,3$. We say that $\varphi(s)$ is $S_{i}$ symmetric if $S_{i}(\varphi(s))=\varphi(s)$. For more details, see Section 4.

The periodic solutions of the differential equation that governs the dynamic of the collinear charged 3-body can be simultaneously $S_{1}$ and $S_{2}$ symmetric, see Section 4. These kind of periodic solutions will be called $S_{12}$-symmetric periodic solutions. In analogous way, we have $S_{13^{-}}$and $S_{23^{-}}$ periodic solutions.

Our results on the $S_{12}$-symmetric periodic solutions for small and positive values of $\mu$ are given in the next theorem.

Theorem 1. Consider $\nu \in(0,1), \alpha>0, \gamma>0, h=h_{1}+h_{2}<0$ and $p$ and $q$ odd positive integers. Then the $S_{12}$-symmetric periodic solutions of the charged 3-body problem (4) for $\mu=0$ and with the initial conditions

(a) $\xi_{1}(0)=0, \xi_{2}(0)=\sqrt{-\gamma / h_{2}}, \eta_{1}(0)=\sqrt{8 \alpha(1-\nu)}, \eta_{2}(0)=0$,

(b) or $\xi_{1}(0)=\sqrt{-\alpha / h_{1}}, \xi_{2}(0)=0, \eta_{1}(0)=0, \eta_{2}(0)=\sqrt{8 \gamma \nu}$,

where $h_{1}=\left(\frac{p \alpha}{q \gamma}\right)^{\frac{2}{3}} h_{2}\left(\frac{1-\nu}{\nu}\right)^{\frac{1}{3}}$, can be continued to a $\mu$-parameter family of $S_{12}$-symmetric periodic orbits of the charged 3-body problem (4) for $\mu>0$ and small.

For the periodic solutions having $S_{13}$-symmetry we obtain the next result.

Theorem 2. Consider $\nu \in(0,1), \alpha>0, \gamma>0, h=h_{1}+h_{2}<0, p$ is odd, $q$ is even positive integers, and either $(p+q-1) / 2$ is even or $(p+q-1) / 2$ is odd and $\alpha \gamma \neq-8(1-\nu) h_{2}^{3}$. Then the $S_{13}$-symmetric periodic solutions of the charged 3-body problem (4) for $\mu=0$ with initial conditions

(a) $\xi_{1}(0)=0, \xi_{2}(0)=\sqrt{-\gamma / h_{2}}, \eta_{1}(0)=\sqrt{8 \alpha(1-\nu)}, \eta_{2}(0)=0$,

(b) or $\xi_{1}(0)=\sqrt{-\alpha / h_{1}}, \xi_{2}(0)=\sqrt{-\gamma / h_{2}}, \eta_{1}(0)=0, \eta_{2}(0)=0$,

where $h_{1}=\left(\frac{p \alpha}{q \gamma}\right)^{\frac{2}{3}} h_{2}\left(\frac{1-\nu}{\nu}\right)^{\frac{1}{3}}$, can be continued to a $\mu$-parameter family

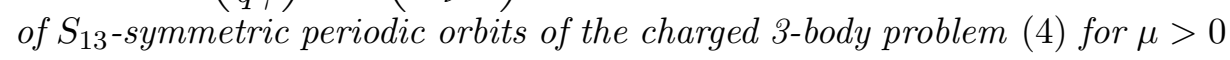
and small.

Finally for the $S_{23}$-symmetric periodic solutions, we get:

Theorem 3. Consider $\nu \in(0,1), \alpha>0, \gamma>0, h=h_{1}+h_{2}<0$, $p$ is even and $q$ is odd positive integers. Then the $S_{23}$-symmetric periodic solutions of the charged 3-body problem (4) for $\mu=0$ with initial conditions

(a) $\xi_{1}(0)=\sqrt{\frac{\alpha}{-h_{1}}}, \xi_{2}(0)=0, \eta_{1}(0)=\sqrt{8 \alpha(1-\nu)}, \eta_{2}(0)=\sqrt{8 \gamma \nu}$,

(b) or $\xi_{1}(0)=\sqrt{\frac{\alpha}{-h_{1}}}, \xi_{2}(0)=\sqrt{\frac{\gamma}{-h_{2}}}, \eta_{1}(0)=0, \eta_{2}(0)=0$, 
where $h_{1}=\left(\frac{p \alpha}{q \gamma}\right)^{\frac{2}{3}} h_{2}\left(\frac{1-\nu}{\nu}\right)^{\frac{1}{3}}$, can be continued to a $\mu$-parameter family of $S_{23}$-symmetric periodic orbits of the charged 3-body problem (4) for $\mu>0$ and small.

\section{Symmetries OF System (4)}

The results obtained in this section are similar to the ones given in [5] for the collinear uncharged three-body system. Consider the involutions

$$
\begin{aligned}
I d:\left(\xi_{1}, \xi_{2}, \eta_{1}, \eta_{2}, s\right) & \mapsto\left(\xi_{1}, \xi_{2}, \eta_{1}, \eta_{2}, s\right), \\
S_{1}:\left(\xi_{1}, \xi_{2}, \eta_{1}, \eta_{2}, s\right) & \mapsto\left(-\xi_{1}, \xi_{2}, \eta_{1},-\eta_{2},-s\right), \\
S_{2}:\left(\xi_{1}, \xi_{2}, \eta_{1}, \eta_{2}, s\right) & \mapsto\left(\xi_{1},-\xi_{2},-\eta_{1}, \eta_{2},-s\right), \\
S_{3}:\left(\xi_{1}, \xi_{2}, \eta_{1}, \eta_{2}, s\right) & \mapsto\left(\xi_{1}, \xi_{2},-\eta_{1},-\eta_{2},-s\right), \\
S_{4}:\left(\xi_{1}, \xi_{2}, \eta_{1}, \eta_{2}, s\right) & \mapsto\left(-\xi_{1},-\xi_{2},-\eta_{1},-\eta_{2}, s\right), \\
S_{5}:\left(\xi_{1}, \xi_{2}, \eta_{1}, \eta_{2}, s\right) & \mapsto\left(-\xi_{1}, \xi_{2},-\eta_{1}, \eta_{2}, s\right), \\
S_{6}:\left(\xi_{1}, \xi_{2}, \eta_{1}, \eta_{2}, s\right) & \mapsto\left(\xi_{1},-\xi_{2}, \eta_{1},-\eta_{2}, s\right), \\
S_{7}:\left(\xi_{1}, \xi_{2}, \eta_{1}, \eta_{2}, s\right) & \mapsto\left(-\xi_{1},-\xi_{2}, \eta_{1}, \eta_{2},-s\right) .
\end{aligned}
$$

A solution $\varphi(s)=\left(\xi_{1}(s), \xi_{2}(s), \eta_{1}(s), \eta_{2}(s)\right)$ of system (4) is invariant under the symmetry $S_{i}$ if $S_{i}(\varphi(s))$ is also a solution of system (4) with $i \in$ $\{1, \ldots, 7\}$. We say that $\varphi(s)$ is $S_{i}$-symmetric if $S_{i}(\varphi(s))=\varphi(s)$.

Note that the set $\left\{I d, S_{1}, \ldots, S_{7}\right\}$ with the usual composition forms an abelian group isomorphic to $\mathbb{Z}_{2} \times \mathbb{Z}_{2} \times \mathbb{Z}_{2}$. This kind of symmetries usually appear in Hamiltonian systems, see for example [3] and [14]. Note that the symmetries $S_{1}, S_{2}$ and $S_{3}$ generate the other ones. In fact,

$$
S_{4}=S_{1} \circ S_{2}, \quad S_{5}=S_{1} \circ S_{3}, \quad S_{6}=S_{2} \circ S_{3}, \quad S_{7}=S_{1} \circ S_{2} \circ S_{3} .
$$

Here we only consider the symmetric periodic orbits with respect to the symmetries $S_{1}, S_{2}$ and $S_{3}$. The periodic solutions of system (4) can be simultaneously $S_{1}$ and $S_{2}$ symmetric. These kind of periodic solutions will be called $S_{12}$-symmetric periodic solutions. In analogous way we have the $S_{13^{-}}$and the $S_{23}$-periodic solutions. Using similar arguments to the ones presented in [3] we can prove the following propositions.

Proposition 1. Consider $\varphi(s)$ a solution of system (4). The following statements hold.

(a) A solution $\varphi(s)$ is a $S_{12}$-symmetric periodic solution of period $S$ if and only if either $\xi_{1}\left(s_{0}\right)=\eta_{2}\left(s_{0}\right)=0$ and $\xi_{2}\left(s_{0}+S / 4\right)=\eta_{1}\left(s_{0}+\right.$ $S / 4)=0$ and there is no $s \in\left(s_{0}, s_{0}+S / 4\right)$ such that $\xi_{2}(s)=\eta_{1}(s)=$ 0 , or $\xi_{2}\left(s_{0}\right)=\eta_{1}\left(s_{0}\right)=0$ and $\xi_{1}\left(s_{0}+S / 4\right)=\eta_{2}\left(s_{0}+S / 4\right)=0$ and there is no $s \in\left(s_{0}, s_{0}+S / 4\right)$ such that $\xi_{1}(s)=\eta_{2}(s)=0$. 
(b) A solution $\varphi(s)$ is a $S_{13}$-symmetric periodic solution of period $S$ if and only if either $\xi_{1}\left(s_{0}\right)=\eta_{2}\left(s_{0}\right)=0$ and $\eta_{1}\left(s_{0}+S / 4\right)=\eta_{2}\left(s_{0}+\right.$ $S / 4)=0$ and there is no $s \in\left(s_{0}, s_{0}+S / 4\right)$ such that $\eta_{1}(s)=\eta_{2}(s)=$ 0 , or $\eta_{1}\left(s_{0}\right)=\eta_{2}\left(s_{0}\right)=0$ and $\xi_{1}\left(s_{0}+S / 4\right)=\eta_{2}\left(s_{0}+S / 4\right)=0$ and there is no $s \in\left(s_{0}, s_{0}+S / 4\right)$ such that $\xi_{1}(s)=\eta_{2}(s)=0$.

(c) A solution $\varphi(s)$ is a $S_{23}$-symmetric periodic solution of period $S$ if and only if either $\xi_{2}\left(s_{0}\right)=\eta_{1}\left(s_{0}\right)=0$ and $\eta_{1}\left(s_{0}+S / 4\right)=\eta_{2}\left(s_{0}+\right.$ $S / 4)=0$ and there is no $s \in\left(s_{0}, s_{0}+S / 4\right)$ such that $\eta_{1}(s)=\eta_{2}(s)=$ 0 or, $\eta_{1}\left(s_{0}\right)=\eta_{2}\left(s_{0}\right)=0$ and $\xi_{2}\left(s_{0}+S / 4\right)=\eta_{1}\left(s_{0}+S / 4\right)=0$ and there is no $s \in\left(s_{0}, s_{0}+S / 4\right)$ such that $\xi_{2}(s)=\eta_{1}(s)=0$.

Furthermore the next result, proved in [5], shows that there are no symmetric periodic solutions having more than two symmetries.

Proposition 2. There are no periodic solutions of system (4) which are

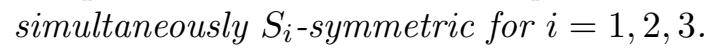

5. Symmetric Periodic orbits of System (4) For $\mu=0$

System (4) with $\mu=0$ is

$$
\begin{aligned}
\frac{d \xi_{1}}{d s} & =\frac{\eta_{1} \xi_{2}^{2}}{1-\nu} \\
\frac{d \xi_{2}}{d s} & =\frac{\eta_{2} \xi_{1}^{2}}{\nu} \\
\frac{d \eta_{1}}{d s} & =\xi_{1}\left(8 \gamma-\frac{\eta_{2}^{2}}{\nu}+8 h \xi_{2}^{2}\right), \\
\frac{d \eta_{2}}{d s} & =\frac{\eta_{1}^{2} \xi_{2}}{\nu-1}+8 \xi_{2}\left(\alpha+h \xi_{1}^{2}\right)
\end{aligned}
$$

with Hamiltonian $G$ given by

$$
G=\frac{1}{2} \xi_{2}^{2}\left(-8 \alpha-\frac{\eta_{1}^{2}}{\nu-1}\right)+\frac{1}{2} \xi_{1}^{2} \xi_{2}^{2}\left(-\frac{8 \gamma}{\xi_{2}^{2}}+\frac{\eta_{2}^{2}}{\nu \xi_{2}^{2}}-8 h\right)
$$

In coordinates $\left(z_{1}, z_{2}, p_{1}, p_{2}\right)$ the Hamiltonian $H$, for $\mu=0$, is given by

$$
H=H_{1}\left(z_{1}, p_{1}\right)+H_{2}\left(z_{2}, p_{2}\right)
$$

where $H_{1}\left(z_{1}, p_{1}\right)=\frac{1}{2}\left(\frac{p_{1}^{2}}{1-\nu}-\frac{2 \alpha}{z_{1}}\right)$ and $H_{2}\left(z_{2}, p_{2}\right)=\frac{1}{2}\left(\frac{p_{2}^{2}}{\nu}-\frac{2 \gamma}{z_{2}}\right)$. Observe that the flow of system (5) on the energy level $H=h$ is given by the flow of the Hamiltonian $H_{1}\left(z_{1}, p_{1}\right)$ on the energy level $H_{1}=h_{1}$, and by the flow of the Hamiltonian $H_{2}\left(z_{2}, p_{2}\right)$ on the energy $H_{2}=h_{2}$ with $h=h_{1}+h_{2}$. 
In the Levi-Civita coordinates the Hamiltonians $H_{1}$ and $H_{2}$ are given by

$$
\begin{array}{ll}
H_{1}\left(\xi_{1}, \eta_{1}\right)=-\frac{\alpha}{\xi_{1}^{2}}-\frac{\eta_{1}^{2}}{8(\nu-1) \xi_{1}^{2}} & =h_{1}, \\
H_{2}\left(\xi_{2}, \eta_{2}\right)=\frac{\eta_{2}^{2}}{8 \nu \xi_{2}^{2}}-\frac{\gamma}{\xi_{2}^{2}} & =h_{2} .
\end{array}
$$

Consider the solution $\varphi(s)=\left(\xi_{1}(s), \xi_{2}(s), \eta_{1}(s), \eta_{2}(s)\right)$ of system (5) satisfying the energy condition $G=0$ (or equivalently $H=h$ ) and we define the new times $\sigma$ and $\tau$ as follows

$$
\begin{array}{llll}
\frac{d \sigma}{d s}=\xi_{2}^{2}, & \text { or equivalently } & \frac{d t}{d \sigma}=4 \xi_{1}^{2}, \\
\frac{d \tau}{d s}=\xi_{1}^{2}, & \text { or equivalently } & \frac{d t}{d \tau}=4 \xi_{2}^{2} .
\end{array}
$$

Note that $\left(\xi_{1}, \eta_{1}\right)$ satisfies the system of differential equations

$$
\begin{aligned}
& \frac{d \xi_{1}}{d \sigma}=\frac{\eta_{1}}{1-\nu}, \\
& \frac{d \eta_{1}}{d \sigma}=8 h_{1} \xi_{1},
\end{aligned}
$$

and $\left(\xi_{2}, \eta_{2}\right)$ satisfies the system of differential equations

$$
\begin{aligned}
\frac{d \xi_{2}}{d \tau} & =\frac{\eta_{2}}{\nu}, \\
\frac{d \eta_{2}}{d \tau} & =8 h_{2} \xi_{2} .
\end{aligned}
$$

Therefore considering the new times $\sigma$ and $\tau$ the functions $G_{1}=G / \xi_{2}^{2}$ and $G_{2}=G / \xi_{1}^{2}$ are

$$
\begin{aligned}
& G_{1}=\frac{8 \alpha(\nu-1)+\eta_{1}^{2}+8 h_{1}(\nu-1) \xi_{1}^{2}}{2(1-\nu)}, \\
& G_{2}=\frac{\eta_{2}^{2}-8 \nu\left(\gamma+h_{2} \xi_{2}^{2}\right)}{2 \nu} .
\end{aligned}
$$

These two functions are the Hamiltonians of system (8) and (9) respectively. Our objective now is to study the periodic solutions of systems (8) and (9). Thus, fixing $h_{1}<0$ we can integrate system (8) directly with the initial conditions $\xi_{1}(0)=\xi_{10}$ and $\eta_{1}(0)=\eta_{10}$, obtaining the solution $\left(\xi_{1}(\sigma), \eta_{1}(\sigma)\right)$ given by

$$
\begin{aligned}
& \xi_{1}(\sigma)=\xi_{10} \cos \left(\omega_{1} \sigma\right)+\frac{\eta_{10}}{\omega_{1}(1-\nu)} \sin \left(\omega_{1} \sigma\right), \\
& \eta_{1}(\sigma)=\eta_{10} \cos \left(\omega_{1} \sigma\right)-\xi_{10} \omega_{1}(1-\nu) \sin \left(\omega_{1} \sigma\right),
\end{aligned}
$$


where $\omega_{1}=\sqrt{\frac{-8 h_{1}}{1-\nu}}$. Note that solution (10) is a periodic solution of system (8) with period $\bar{\sigma}=2 \pi / \omega_{1}$. Assuming that solution (10) satisfies the energy relation $G_{1}=0$ we obtain the following relationship between the initial conditions $\xi_{10}$ and $\eta_{10}$ :

$$
-4 \alpha+\frac{\eta_{10}^{2}}{2-2 \nu}-4 h_{1} \xi_{10}^{2}=0 .
$$

Moreover by the parametrization of the time given in (7) we obtain the period of this solution in terms of the time $t$, i.e.

$$
T_{1}\left(h_{1}, \alpha, \nu\right)=\int_{0}^{\bar{\sigma}} 4 \xi_{1}^{2}(\sigma) d \sigma=\frac{\sqrt{2(1-\nu)} \pi \alpha}{h_{1} \sqrt{-h_{1}}} .
$$

Similarly fixing $h_{2}<0$ we can integrate system (9) with the initial conditions $\xi_{2}(0)=\xi_{20}$ and $\eta_{2}(0)=\eta_{20}$, getting the solution $\left(\xi_{2}(\tau), \eta_{2}(\tau)\right)$ given by

$$
\begin{aligned}
& \xi_{2}(\tau)=-\xi_{20} \cos \left(\omega_{2} \tau\right)+\frac{\eta_{20}}{\omega_{2} \nu} \sin \left(\omega_{2} \tau\right), \\
& \eta_{2}(\tau)=\eta_{20} \cos \left(\omega_{2} \tau\right)+\xi_{20} \omega_{2} \nu \sin \left(\omega_{2} \tau\right),
\end{aligned}
$$

where $\omega_{2}=\sqrt{\frac{-8 h_{2}}{\nu}}$. Solution (12) is periodic with period $\bar{\tau}=2 \pi / \omega_{2}$. Assuming that solution (12) satisfies the energy relation $G_{2}=0$, the initial conditions $\xi_{20}$ and $\eta_{20}$ satisfy the equation

$$
\frac{\eta_{20}^{2}-8 \nu\left(\gamma+h_{2} \xi_{20}^{2}\right)}{2 \nu}=0,
$$

and by (7) the period of solution (12) in time $t$ is given by

$$
T_{2}\left(h_{2}, \gamma, \nu\right)=\int_{0}^{\bar{\tau}} 4 \xi_{2}^{2}(\tau) d \tau=\frac{\pi \gamma \sqrt{-2 \nu h_{2}}}{h_{2}^{2}} .
$$

In the following we summarize the relationship between the times $\sigma, \tau, t$ and $S$ :

\begin{tabular}{|c|c|c|c|c|}
\hline Time & $\sigma$ & $\tau$ & $t$ & $s$ \\
\hline Period & $\sigma^{*}=p \bar{\sigma}$ & $\tau^{*}=q \bar{\tau}$ & $T=p T_{1}\left(h_{1}, \alpha, \nu\right)=q T_{2}\left(h_{2}, \gamma, \nu\right)$ & $S^{*}=s(T)$ \\
\hline Period $/ 4$ & $\sigma^{*} / 4$ & $\tau^{*} / 4$ & $T / 4$ & $S^{*} / 4$ \\
\hline
\end{tabular}

TABLE 1. Period of the solution $\varphi(s)$ of $(5)$.

Proposition 3. Consider the periodic solutions $\left(\xi_{1}(\sigma), \eta_{1}(\sigma)\right)$ the $\left(\xi_{2}(\tau), \eta_{2}(\tau)\right)$ of systems (8) and (9) with periods $\bar{\sigma}$ and $\bar{\tau}$, satisfying the energy conditions $G_{1}=0$ and $G_{2}=0$, respectively. Assume that the functions $\sigma(s)$ and $\tau(s)$ given in (7) satisfy $\sigma(0)=\tau(0)=0$ and there is no $s \in \mathbb{R}$ such that $\xi_{1}(\sigma(s))=\xi_{2}(\tau(s))=0$. Then the following statements holds. 
(a) The solution $\varphi(s)=\left(\xi_{1}(\sigma(s)), \xi_{2}\left(\tau(s), \eta_{1}\left(\sigma(s), \eta_{2}(\tau(s))\right.\right.\right.$ of system (5) with initial condition $\xi_{1}(0)=\xi_{10}, \xi_{2}(0)=\xi_{20}, \eta_{1}(0)=\eta_{10}, \eta_{2}(0)=$ $\eta_{20}$ satisfies the energy relation $G\left(\xi_{10}, \xi_{20}, \eta_{10}, \eta_{20}\right)=0$.

(b) If $h_{1}=\left(\frac{p \alpha}{q \gamma}\right)^{\frac{2}{3}} h_{2}\left(\frac{1-\nu}{\nu}\right)^{\frac{1}{3}}$ for some $p, q \in \mathbb{N}$ coprime, then $\varphi(s)$ is a periodic solution of system (5).

(c) Let $s(t)$ be the inverse function of

$$
t(s)=\int_{0}^{s} 4 \xi_{1}^{2}(\theta) \xi_{2}^{2}(\theta) d \theta .
$$

For the $h_{1}$ given in $(b)$ the period and the quarter of period in times $\sigma, \tau, t$ and $s$ are given in Table 1.

Proof. The proof of the statement (a) follows by the definition of $\left(\xi_{1}(\sigma), \eta_{1}(\sigma)\right)$, $\left(\xi_{2}(\tau), \eta_{2}(\tau)\right)$ together with the fact that the initial conditions satisfy the equations (11) and (13).

Note that when we consider the time $t$ the periodic solutions $\left(\xi_{1}(\sigma), \eta_{1}(\sigma)\right)$ and $\left(\xi_{2}(\tau), \eta_{2}(\tau)\right)$ possess periods $T_{1}\left(h_{1}, \alpha, \nu\right)$ and $T_{2}\left(h_{2}, \gamma, \nu\right)$, respectively. Therefore, there exist a periodic solution of system (5) because

$$
p T_{1}\left(h_{1}, \alpha, \nu\right)=q T_{2}\left(h_{2}, \gamma, \nu\right),
$$

for some $p, q \in \mathbb{N}$ coprime. Solving equation (14) we obtain the expression given in statement $(b)$. Note that the time $t=T / 4$ corresponds to the time $\sigma=\sigma^{*} / 4$, and similarly for the times $\tau=\tau^{*} / 4$ and $s=S^{*} / 4$. Furthermore system (8) is invariant under the symmetry

$$
\left(\xi_{1}, \eta_{1}, \sigma\right) \mapsto\left(-\xi_{1}, \eta_{1},-\sigma\right) .
$$

Therefore the function $\xi_{1}$ satisfies $\xi_{1}(\sigma)=-\xi_{1}(-\sigma)$, i.e., $\xi_{1}^{2}(\sigma)$ is an even function. Therefore the period of $\xi_{1}^{2}$ is given by $\bar{\sigma} / 2$ and we get

$$
T_{1}=\int_{0}^{\bar{\sigma}} 4 \xi_{1}^{2}(\sigma) d \sigma=2 \int_{0}^{\bar{\sigma} / 2} 4 \xi_{1}^{2}(\sigma) d \sigma=4 \int_{0}^{\bar{\sigma} / 4} 4 \xi_{1}^{2}(\sigma) d \sigma .
$$

On the other hand we have

$$
t\left(\sigma^{*} / 4\right)=\int_{0}^{p \bar{\sigma} / 4} 4 \xi_{1}^{2}(\sigma) d \sigma=p \int_{0}^{\bar{\sigma} / 4} 4 \xi_{1}^{2}(\sigma) d \sigma=p \frac{T_{1}}{4}=\frac{T}{4} .
$$

Therefore the time $t=T / 4$ corresponds to the time $\sigma=\sigma^{*} / 4$. Analogously we get that $t=T / 4$ corresponds to the time $\tau=\tau^{*} / 4$ and statement $(c)$ is proved.

Observe that by statement $(c)$ of Proposition 3 we have that $d t / d s>0$ when there are no collisions, and zero in the binary collisions. Therefore the inverse function $s=s(t)$ exists always that the system has no triple collision, and it is differentiable if there is no binary collisions. As in the uncharged case studied in [5], page 128, the number $p$ in Proposition 3 represents the 
number of binary collisions between $m_{1}$ and $m_{2}$, and the number $q$ the binary collisions between the particles $m_{2}$ and $m_{3}$.

We stress that the main objective of this paper is to analyse the periodic orbits of system (4) satisfying the energy relation $G=0$. So the next proposition provides the initial conditions in order to prove that the solutions of system (4) are symmetric. Consider the initial conditions given in Proposition 3.

Proposition 4. The following statements hold.

(a) If $p$ and $q$ are odd then the solution $\varphi(s)$ given in Proposition 3 with initial conditions

$$
\begin{array}{ll}
\text { either } & \xi_{10}=0, \xi_{20}^{*}=\sqrt{\frac{\gamma}{-h_{2}}}, \eta_{10}^{*}=\sqrt{8 \alpha(1-\nu)}, \eta_{20}=0, \\
\text { or } & \xi_{10}^{*}=\sqrt{\frac{\alpha}{-h_{1}}}, \xi_{20}=0, \eta_{10}=0, \eta_{20}^{*}=\sqrt{8 \gamma \nu},
\end{array}
$$

is a $S_{12}$-symmetric periodic solution.

(b) If $p$ is odd and $q$ is even then the solution $\varphi(s)$ given in Proposition 3 with initial conditions

$$
\begin{array}{ll}
\text { either } & \xi_{10}^{*}=0, \xi_{20}^{*}=\sqrt{\frac{\gamma}{-h_{2}}}, \eta_{10}^{*}=\sqrt{8 \alpha(1-\nu)}, \eta_{20}^{*}=0, \\
\text { or } \quad \xi_{10}^{*}=\sqrt{\frac{\alpha}{-h_{1}}}, \xi_{20}^{*}=\sqrt{\frac{\gamma}{-h_{2}}}, \eta_{10}^{*}=0, \eta_{20}^{*}=0,
\end{array}
$$

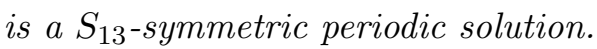

(c) If $p$ is even and $q$ is odd then the solution $\varphi(s)$ given in Proposition 3 with initial conditions

$$
\begin{array}{ll}
\text { either } & \xi_{10}^{*}=\sqrt{\frac{\alpha}{-h_{1}}}, \xi_{20}^{*}=0, \eta_{10}^{*}=0, \eta_{20}^{*}=\sqrt{8 \gamma \nu}, \\
\text { or } & \xi_{10}^{*}=\sqrt{\frac{\alpha}{-h_{1}}}, \xi_{20}^{*}=\sqrt{\frac{\gamma}{-h_{2}}}, \eta_{10}^{*}=0, \eta_{20}^{*}=0,
\end{array}
$$

is a $S_{23}$-symmetric periodic solution.

Proof. Consider the solution $\varphi(s)=\left(\xi_{1}(\sigma(s)), \xi_{2}(\tau(s)), \eta_{1}(\sigma(s)), \eta_{2}(\tau(s))\right)$ of system (4). The proof follows evaluating $\varphi(s)$ at times $s=0$ and $s=S^{*} / 4$, as given in Table 1.

Remark 1. Note that the Levi-Civita transformation duplicates the number of orbits. Hence it is sufficient to consider the positive square roots of the initial conditions given in Proposition 4. 


\section{The COnTINuAtion Method And The Symmetric PERIODiC SOLUTIONS}

For the initial conditions provided in Proposition 4 we have $S_{12^{-}}, S_{13^{-}}$, $S_{23}$-symmetric periodic orbits for system (4) when $\mu=0$. To provide symmetric periodic orbits for small positive values of $\mu$ we apply the continuation method of Poincaré.

6.1. $S_{12}$-symmetric periodic solutions. By Proposition 4 the solution $\varphi\left(s ; \xi_{10}, \xi_{20}, \eta_{10}, \eta_{20},(\alpha, \beta, \gamma, \nu, \mu)\right)$ of system (4) for $\mu=0$ is a $S_{12}$-symmetric if the initial conditions satisfies

(a) either $\xi_{1}(0)=0, \xi_{2}(0)=\xi_{20}^{*}, \eta_{1}(0)=\eta_{10}^{*}, \eta_{2}(0)=0$;

(b) or $\xi_{1}(0)=\xi_{10}^{*}, \xi_{2}(0)=0, \eta_{1}(0)=0, \eta_{2}(0)=\eta_{20}^{*}$;

for fixed values of $\alpha, \beta, \gamma, \nu$ and $h$.

6.1.1. Case (a). By statement (a) of Proposition 1 we have that the solution $\varphi$ is a $S_{12}$-symmetric periodic solution of the charged collinear 3-body problem with period $S$ satisfying the energy condition $G=0$ if and only if

$$
\begin{array}{ll}
\xi_{2}\left(S / 4 ; \xi_{20}, \eta_{10},(\alpha, \beta, \gamma, \mu)\right) & =0, \\
\eta_{1}\left(S / 4 ; \xi_{20}, \eta_{10},(\alpha, \beta, \gamma, \mu)\right) & =0, \\
G\left(\xi_{20}, \eta_{10},(\alpha, \beta, \gamma, \mu)\right) & =0 .
\end{array}
$$

The solution of the last equation in terms of $\eta_{10}$ is given by

$$
\eta_{10}=\sqrt{\frac{8 \alpha(1-\mu)(1-\nu)}{1-\mu \nu}} .
$$

Thus the solution $\varphi\left(s ; 0, \xi_{20}, \eta_{10}, 0,(\alpha, \beta, \gamma, \nu, \mu)\right)$ is a $S_{12}$-symmetric periodic solution of system (4) that satisfies $G=0$ if and only if

$$
\begin{aligned}
& \xi_{2}\left(S / 4 ; \xi_{20},(\alpha, \beta, \gamma, \mu)\right)=0, \\
& \eta_{1}\left(S / 4 ; \xi_{20},(\alpha, \beta, \gamma, \mu)\right)=0 .
\end{aligned}
$$

Statement $(a)$ of Proposition 4 provides additional information on the initial conditions and on the numbers $p$ and $q$, see Table 1 , for these symmetric periodic solutions. Thus if $p=2 m+1, q=2 k+1, S=S^{*}=s\left(p T_{1}\left(h_{1}, \alpha\right)\right)=$ $s\left(q T_{2}\left(h_{2}, \gamma\right)\right), \xi_{20}=\sqrt{\frac{\gamma}{-h_{2}}}$ and $\eta_{10}=\sqrt{8 \alpha(1-\nu)}$, with $m$ and $k$ positive integers, then $\varphi\left(s ; 0, \xi_{20}, \eta_{10}, 0,(\alpha, \beta, \gamma, \nu, 0)\right)$ is a $S_{12}$-symmetric solution of system (4) for $\mu=0$ and the energy level $H=h=h_{1}+h_{2}$ satisfies the condition $h_{1}=\left(\frac{p \alpha}{q \gamma}\right)^{\frac{2}{3}} h_{2}\left(\frac{1-\nu}{\nu}\right)^{\frac{1}{3}}$ given in statement $(b)$ of Proposition 3. Our objective now is to extend this solution to $\mu>0$ and small. Applying 
the Implicit Function Theorem to system (15) in a neighbourhood of a known solution we have that if

$$
\left|\begin{array}{ll}
\frac{\partial \xi_{2}}{\partial s} & \frac{\partial \xi_{2}}{\partial \xi_{20}} \\
\frac{\partial \eta_{1}}{\partial s} & \frac{\partial \eta_{1}}{\partial \xi_{20}}
\end{array}\right| \mid \begin{array}{ll}
s & =S^{*} / 4 \\
\xi_{20} & =\xi_{20}^{*} \\
\mu & =0
\end{array} \quad \neq 0
$$

then there exist unique analytic functions $\xi_{20}=\xi_{20}(\mu)$ and $S=S(\mu)$ defined for $\mu \geq 0$ sufficiently small that satisfy

(i) $\xi_{20}(0)=\xi_{20}^{*}$ and $S(0)=S^{*}$,

(ii) and $\varphi\left(s ; 0, \xi_{20}, \eta_{10}, 0, \mu\right)$ is a $S_{12}$-symmetric periodic solution of (4) with period $S=S(\mu)$ satisfying the energy condition $G=0$.

Note that the derivatives $\partial \xi_{2} / \partial s$ and $\partial \eta_{1} / \partial s$ are obtained evaluating the right hand of system (4) for $\mu=0, s=S^{*} / 4$ and with the initial conditions $\left(0, \xi_{20}^{*}, \eta_{10}, 0\right)$. So

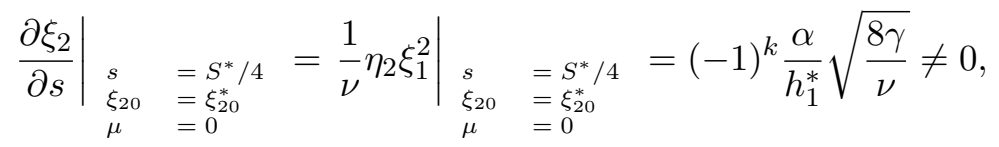

and

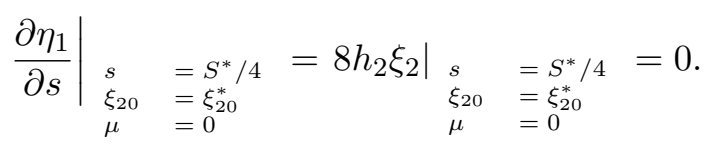

Therefore it remains to calculate $\partial \eta_{1} / \partial \xi_{20}$ evaluated at $s=S^{*} / 4, \xi_{20}=\xi_{20}^{*}$ and $\mu=0$. We obtain this value derivating the solution of system (5)

$$
\eta_{1}\left(\sigma(s) ; 0, \xi_{20}, \eta_{10}, 0\right),
$$

with respect to the variable $\xi_{20}$, where the initial conditions $\xi_{1}(0)=0, \xi_{2}(0)=$ $\xi_{20}, \eta_{1}(0)=\eta_{10}, \eta_{2}(0)=0$ satisfy the energy relation $G=0$. So

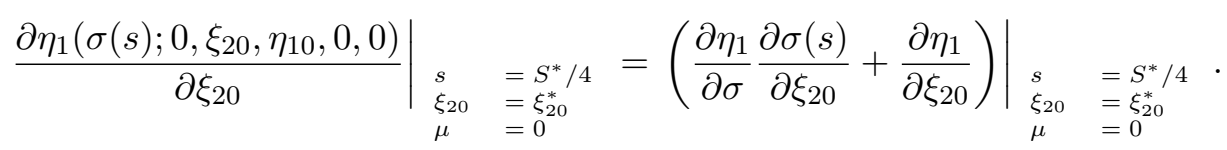

By (7) we get the relationship between $\xi_{20}, \tau$ and $\sigma$

$$
\xi_{1}^{2}(\sigma) d \sigma=\xi_{2}^{2}(\tau) d \tau
$$

Integrating this equation and assuming that $\sigma(0)=0$ and $\tau(0)=0$ we obtain the relation between $\sigma(s)$ and $\tau(s)$, i.e.

$$
\begin{aligned}
& \frac{\alpha(1-\mu)}{h_{1}^{2}(1-\mu \nu)}\left(\sqrt{-2 h_{1}(1-\nu)} \sin \left(2 \omega_{1} \sigma(s)\right)-8 h_{1} \sigma(s)\right) \\
& -\xi_{20}^{2}\left(-\frac{\sqrt{2 \nu} \sin \left(2 \omega_{2} \tau(s)\right)}{\sqrt{-h_{2}}}+8 \tau(s)\right)=0
\end{aligned}
$$


where $\omega_{1}=\sqrt{\frac{-8 h_{1}}{1-\nu}}, \omega_{2}=\sqrt{\frac{-8 h_{2}}{\nu}}, h_{2}=-\frac{\gamma}{\xi_{20}^{2}}$ and $h_{1}=h-h_{2}$. Derivating implicitly equation (17) with respect to the variable $\xi_{20}$ we obtain

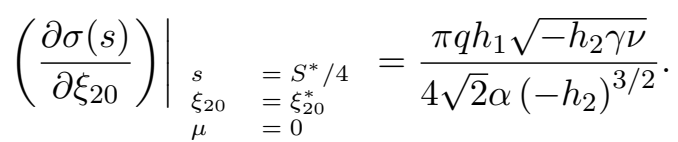

From (8) and (10) we get

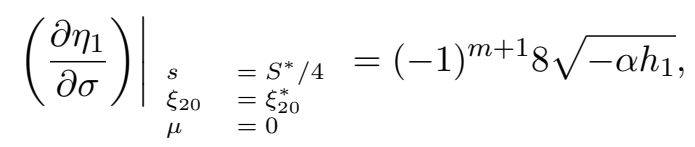

and

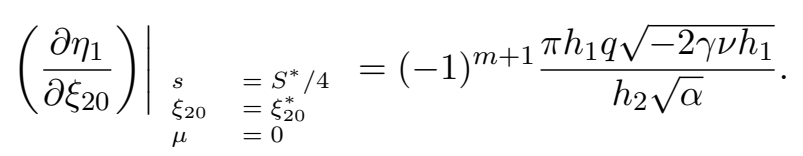

So

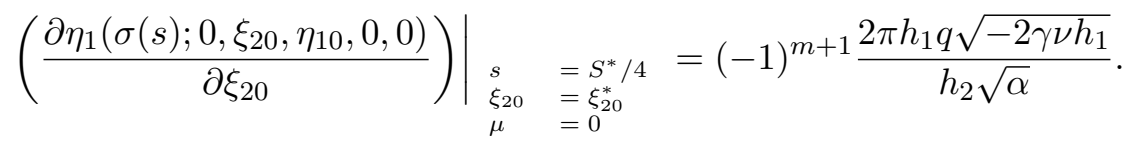

Finally the determinant (16) is given by

$$
(-1)^{k+m}(2 k+1) \frac{8 \pi \gamma \sqrt{-\alpha h_{1}}}{h_{2}}
$$

Therefore we conclude that the determinant is zero if and only if $\alpha \gamma=0$.

6.1.2. Case (b). As in the previous case, from the second part of statement (a) of Proposition 1 we have that the solution $\varphi$ is a $S_{12}$-symmetric periodic solution with period $S$ satisfying the energy condition $G=0$ if and only if

$$
\begin{array}{ll}
\xi_{1}\left(S / 4 ; \xi_{10}, \eta_{20},(\alpha, \beta, \gamma, \mu)\right) & =0, \\
\eta_{2}\left(S / 4 ; \xi_{10}, \eta_{20},(\alpha, \beta, \gamma, \mu)\right) & =0, \\
G\left(\xi_{10}, \eta_{20},(\alpha, \beta, \gamma, \mu)\right) & =0 .
\end{array}
$$

The solution of $G=0$ in terms of $\eta_{20}$ is given by

$$
\eta_{20}=\frac{\sqrt{8 \gamma \nu(1-\mu)}}{\sqrt{\mu \nu-\mu+1}}
$$

So the solution $\varphi\left(s ; \xi_{10}, 0,0, \eta_{20},(\alpha, \beta, \gamma, \nu, \mu)\right)$ is a $S_{12}$-symmetric periodic solution of system (4) that satisfies $G=0$ if and only if

$$
\begin{aligned}
& \xi_{1}\left(S / 4 ; \xi_{10},(\alpha, \beta, \gamma, \mu)\right)=0, \\
& \eta_{2}\left(S / 4 ; \xi_{10},(\alpha, \beta, \gamma, \mu)\right)=0 .
\end{aligned}
$$

Again by statement $(a)$ of Proposition 4 we obtain the initial conditions and the numbers $p$ and $q$ to have symmetric periodic solutions (see Table 1 ), i.e. $p=2 m+1, q=2 k+1, S=S^{*}=s\left(p T_{1}\left(h_{1}, \alpha\right)\right)=s\left(q T_{2}\left(h_{2}, \gamma\right)\right), \xi_{10}^{*}=$ 
$\sqrt{\frac{\alpha}{-h_{1}}}, \eta_{20}^{*}=\sqrt{8 \gamma \nu}$, for $\mu=0$ and the energy level $H=h=h_{1}+h_{2}$ satisfies the condition $h_{1}=\left(\frac{p \alpha}{q \gamma}\right)^{\frac{2}{3}} h_{2}\left(\frac{1-\nu}{\nu}\right)^{\frac{1}{3}}$ given in statement $(b)$ of Proposition 3. As in the previous case applying the Implicit Function Theorem to system (22) in a neighborhood of a known solution we have that if

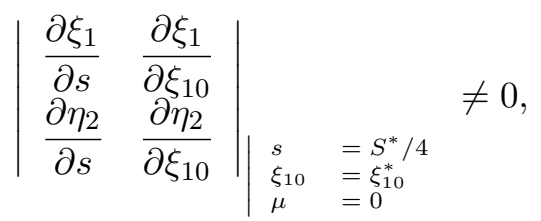

then there exist unique analytic functions $\xi_{10}=\xi_{10}(\mu)$ and $S=S(\mu)$ defined for $\mu \geq 0$ sufficiently small that satisfies

(i) $\xi_{10}(0)=\xi_{10}^{*}$ and $S(0)=S^{*}$,

(ii) and $\varphi\left(s ; \xi_{10}, 0,0, \eta_{20}, \mu\right)$ is a $S_{12}$-symmetric periodic solution of system (4) with period $S=S(\mu)$ satisfying the energy condition $G=0$.

In the same way as we work in the previous case we obtain

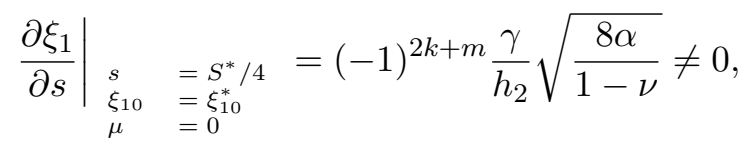

and

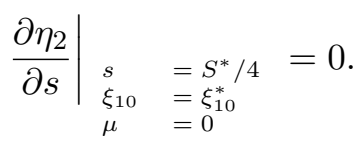

So it remains to calculate $\partial \eta_{2} / \partial \xi_{10}$ evaluated at $s=S^{*} / 4, \xi_{10}=\xi_{10}^{*}$ and $\mu=$ 0 . This derivative is obtained derivating the solution $\eta_{2}\left(\tau(s) ; \xi_{10}, 0,0, \eta_{20}, 0\right)$ of system (5) evaluated in $\xi_{10}=\xi_{10}^{*}, \xi_{2}(0)=0, \eta_{1}(0)=0, \eta_{2}(0)=\eta_{20}, s=$ $S^{*} / 4$ and satisfying the energy relation $G=0$. Then

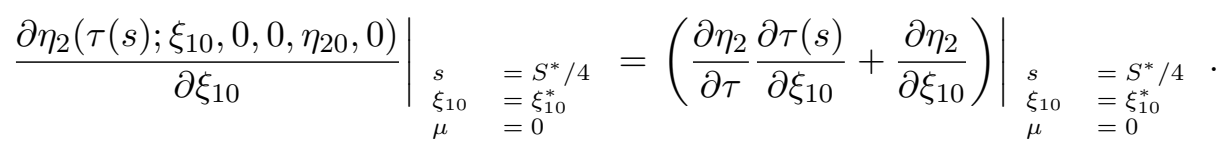

As in the previous case we obtain

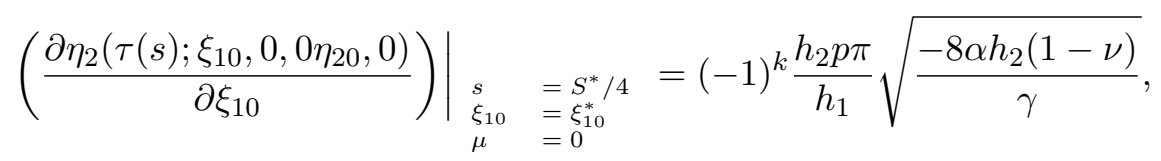

and the determinant (19) is given by

$$
(-1)^{k+m+1} \frac{8 p \pi \alpha}{h_{1}} \sqrt{-\gamma h_{2}} .
$$

Therefore we conclude that this determinant is zero if and only if $\alpha \gamma=0$ and Theorem 1 is proved. 
6.2. $S_{13}$-symmetric periodic orbits. By Proposition 4 the solution $\varphi\left(s ; \xi_{10}\right.$, $\left.\xi_{20}, \eta_{10}, \eta_{20},(\alpha, \beta, \gamma, \nu, \mu)\right)$ of system (4) for $\mu=0$ is a $S_{13}$-symmetric if the initial conditions satisfies

(a) either $\xi_{1}(0)=0, \xi_{2}(0)=\xi_{20}^{*}, \eta_{1}(0)=\eta_{10}^{*}, \eta_{2}(0)=0$;

(b) or $\xi_{1}(0)=\xi_{10}^{*}, \xi_{2}(0)=\xi_{20}^{*}, \eta_{1}(0)=0, \eta_{2}(0)=0$;

for fixed values of $\alpha, \beta, \gamma, \nu$ and $h$.

6.2.1. Case (a). By statement (b) of Proposition 1 we have that the solution $\varphi$ is a $S_{13}$-symmetric periodic solution of the charged collinear 3-body problem with period $S$ satisfying the energy condition $G=0$ if and only if

$$
\begin{array}{ll}
\eta_{1}\left(S / 4 ; \xi_{20}, \eta_{10},(\alpha, \beta, \gamma, \mu)\right) & =0, \\
\eta_{2}\left(S / 4 ; \xi_{20}, \eta_{10},(\alpha, \beta, \gamma, \mu)\right) & =0, \\
G\left(\xi_{20}, \eta_{10},(\alpha, \beta, \gamma, \mu)\right) & =0 .
\end{array}
$$

Solving the last equation in terms of $\eta_{10}$ we obtain

$$
\eta_{10}=\sqrt{\frac{8 \alpha(1-\mu)(1-\nu)}{1-\mu \nu}} .
$$

Then the solution $\varphi\left(s ; 0, \xi_{20}, \eta_{10}, 0,(\alpha, \beta, \gamma, \nu, \mu)\right)$ is a $S_{12}$-symmetric periodic solution of system (4) that satisfies $G=0$ if and only if

$$
\begin{aligned}
& \eta_{1}\left(S / 4 ; \xi_{20},(\alpha, \beta, \gamma, \mu)\right)=0, \\
& \eta_{2}\left(S / 4 ; \xi_{20},(\alpha, \beta, \gamma, \mu)\right)=0 .
\end{aligned}
$$

Statement $(b)$ of Proposition 4 provides the initial conditions and numbers $p$ and $q$, see Table 1 in order that these periodic orbits are symmetric, i.e. $p=$ $2 m+1, q=2 k, S=S^{*}=s\left(p T_{1}\left(h_{1}, \alpha\right)\right)=s\left(q T_{2}\left(h_{2}, \gamma\right)\right), \xi_{20}=\sqrt{\frac{\gamma}{-h_{2}}}$ and $\eta_{10}=\sqrt{8 \alpha(1-\nu)}$, with $m$ and $k$ positive integers, then $\varphi\left(s ; 0, \xi_{20}, \eta_{10}, 0,(\alpha, \beta\right.$, $\gamma, \nu, 0)$ ) is a $S_{13}$-symmetric solution for system (4) for $\mu=0$. Moreover, the energy level $H=h=h_{1}+h_{2}$ satisfies the condition $h_{1}=\left(\frac{p \alpha}{q \gamma}\right)^{\frac{2}{3}} h_{2}\left(\frac{1-\nu}{\nu}\right)^{\frac{1}{3}}$ given in statement $(b)$ of Proposition 3. Our objective now is to extend this solution for $\mu>0$ and small. Applying the Implicit Function Theorem in system (20) in a neighborhood of a known solution we have that if

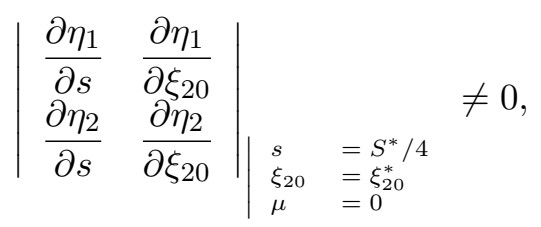

then there exist unique analytic functions $\eta_{20}=\eta_{20}(\mu)$ and $S=S(\mu)$ defined for $\mu \geq 0$ sufficiently small that satisfies 
(i) $\eta_{20}(0)=0$ and $S(0)=S^{*}$,

(ii) and $\varphi\left(s ; 0, \xi_{20}, \eta_{10}, 0, \mu\right)$ is a $S_{13}$-symmetric periodic solution of system (4) with period $S=S(\mu)$ satisfying the energy condition $G=0$.

As in the previous case we get that

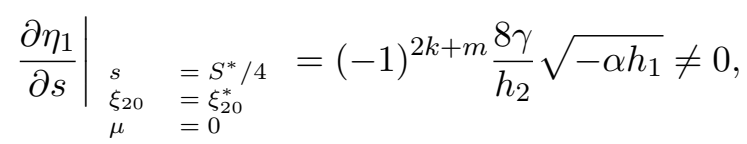

and

$$
\left.\frac{\partial \eta_{2}}{\partial s}\right|_{\substack{s \\ \xi_{20} \\ \mu}}=S_{\xi_{20}^{*}}^{*} / 4=(-1)^{k+2 m+1} \frac{8 \alpha}{h_{1}} \sqrt{-\gamma h_{2}} \neq 0 .
$$

Now we have to calculate the other two derivatives $\partial \eta_{1} / \partial \xi_{20}$ and $\partial \eta_{2} / \partial \xi_{20}$ evaluated at $s=S^{*} / 4, \xi_{20}=\xi_{20}^{*}$ and $\mu=0$. We obtain these values derivating the solutions $\eta_{1}\left(\sigma(s) ; 0, \xi_{20}, \eta_{10}, 0\right)$ and $\eta_{2}\left(\tau(s) ; 0, \xi_{20}, \eta_{10}, 0\right)$ of system (5) with respect to the variable $\xi_{20}$, where the initial conditions $\xi_{1}(0)=0, \xi_{2}(0)=\xi_{20}, \eta_{1}(0)=\eta_{10}, \eta_{2}(0)=0$ satisfy the energy relation $G=0$. So

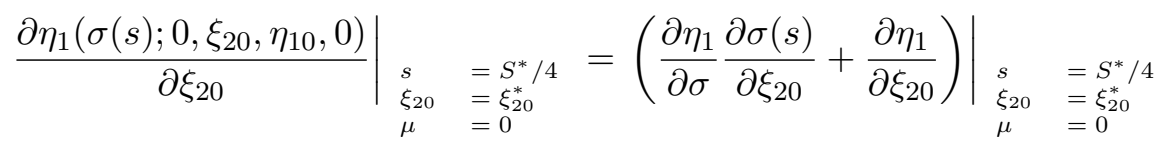

is

$$
\frac{(-1)^{m+1} 4 h_{1}^{2}}{h_{2}}(1-\nu) \sqrt{-\alpha h_{1}}\left[\pi q \sqrt{2 \gamma \nu}+4 \gamma \frac{\partial \tau}{\partial s}\left(\sqrt{-\gamma / h_{2}}\right)\right],
$$

and

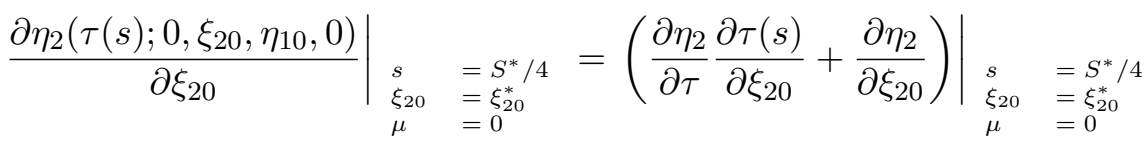

is

$$
(-1)^{k} 16 \sqrt{-\gamma h_{2}} \frac{\partial \tau}{\partial s}\left(\sqrt{-\gamma / h_{2}}\right)
$$

Therefore determinant (21) is given by

$$
(-1)^{k+m} 16 \pi q \gamma \sqrt{\frac{2 \alpha \nu h_{1}}{h_{2}}} .
$$

Hence we conclude that the determinant is zero if and only if $\alpha \gamma=0$.

6.2.2. Case (b). From the second part of statement (b) of Proposition 1 the solution $\varphi$ is a $S_{13}$-symmetric periodic solution with period $S$ satisfying the energy condition $G=0$ if and only if

$$
\begin{array}{ll}
\xi_{1}\left(S / 4 ; \xi_{10}, \xi_{20},(\alpha, \beta, \gamma, \mu)\right) & =0, \\
\eta_{2}\left(S / 4 ; \xi_{10}, \xi_{20},(\alpha, \beta, \gamma, \mu)\right) & =0, \\
G\left(\xi_{10}, \xi_{20},(\alpha, \beta, \gamma, \mu)\right) & =0 .
\end{array}
$$


Therefore the solution of the equation $G=0$ in terms of $\xi_{20}$ is given by

$$
\xi_{20}=\sqrt{\frac{\alpha h_{2}-h_{1}(\gamma+\beta \mu)+\sqrt{\left(\alpha h_{2}\right)^{2}+2 h_{1} h_{2} \alpha(\gamma-\beta \mu)+h_{1}^{2}(\gamma+\beta \mu)^{2}}}{2 h_{1} h_{2}}} .
$$

So the solution $\varphi\left(s ; \xi_{10}, 0,0, \eta_{20},(\alpha, \beta, \gamma, \nu, \mu)\right)$ is a $S_{13}$-symmetric periodic solution of system (4) that satisfies $G=0$ if and only if

$$
\begin{aligned}
& \xi_{1}\left(S / 4 ; \xi_{10},(\alpha, \beta, \gamma, \mu)\right)=0, \\
& \eta_{2}\left(S / 4 ; \xi_{10},(\alpha, \beta, \gamma, \mu)\right)=0 .
\end{aligned}
$$

Again by statement $(b)$ of Proposition 4 we have the initial conditions and the numbers $p$ and $q$ (see Table 1 ) in order to the solutions are symmetric, i.e. $p=2 m+1, q=2 k, S=S^{*}=s\left(p T_{1}\left(h_{1}, \alpha\right)\right)=s\left(q T_{2}\left(h_{2}, \gamma\right)\right), \xi_{10}^{*}=$ $\sqrt{\frac{\alpha}{-h_{1}}}, \eta_{20}^{*}=\sqrt{8 \gamma \nu}$, for $\mu=0$. Furthermore the energy level $H=h=$ $h_{1}+h_{2}$ satisfies the condition $h_{1}=\left(\frac{p \alpha}{q \gamma}\right)^{\frac{2}{3}} h_{2}\left(\frac{1-\nu}{\nu}\right)^{\frac{1}{3}}$ given in statement (b) of Proposition 3. As in the previous case applying the Implicit Function Theorem to system (22) in a neighbourhood of a known solution we have that if

$$
\left|\begin{array}{cc}
\frac{\partial \xi_{1}}{\partial s} & \frac{\partial \xi_{1}}{\partial \xi_{10}} \\
\frac{\partial \eta_{2}}{\partial s} & \frac{\partial \eta_{2}}{\partial \xi_{10}}
\end{array}\right|_{\substack{s \\
\begin{array}{l}
s \\
\xi_{10} \\
\mu
\end{array} \\
=\xi_{10}^{*} \\
=0}} \neq 0
$$

then there exist unique analytic functions $\xi_{10}=\xi_{10}(\mu)$ and $S=S(\mu)$ defined for $\mu \geq 0$ sufficiently small that satisfy

(i) $\xi_{10}(0)=\xi_{10}^{*}$ and $S(0)=S^{*}$,

(ii) and $\varphi\left(s ; \xi_{10}, 0,0, \eta_{20}, \mu\right)$ is a $S_{13}$-symmetric periodic solution of system (4) with period $S=S(\mu)$ satisfying the energy condition $G=0$.

Analogously to the previous case we obtain

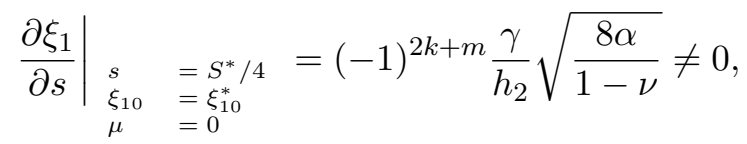

and

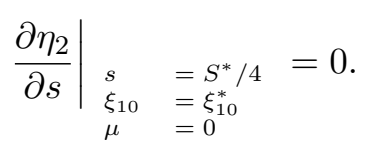

So it remains to calculate $\partial \eta_{2} / \partial \xi_{10}$ evaluated at $s=S^{*} / 4, \xi_{10}=\xi_{10}^{*}$ and $\mu=$ 0 . This derivative is obtained by derivating the solution $\eta_{2}\left(\tau(s) ; \xi_{10}, \xi_{20}, 0,0,0\right)$ of system (5) evaluated in $\xi_{10}=\xi_{10}^{*}$ and $s=S^{*} / 4$ with initial conditions

$$
\xi_{10}=\xi_{10}, \xi_{2}(0)=\xi_{20}, \eta_{1}(0)=0, \eta_{2}(0)=0
$$


satisfying the energy relation $G=0$. Then

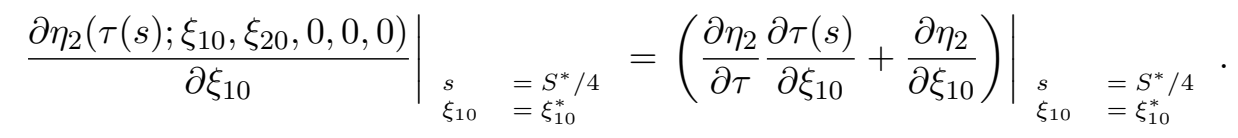

As in the previous case we obtain that

is

$$
\left.\frac{\partial \eta_{2}\left(\tau(s) ; \xi_{10}, \xi_{20}, 0,0,0\right)}{\partial \xi_{10}}\right|_{\substack{s \\ \xi_{10}}=S_{10}^{*} / 4}
$$

$$
\frac{(-1)^{k} p \pi}{2 \gamma h_{1}}\left((-1)^{k+m} \alpha \gamma-\sqrt{-8 \alpha \gamma(1-\nu) h_{2}^{3}}\right),
$$

and determinant (23) is given by

$$
(-1)^{k+m+1} \frac{\sqrt{2} p \pi \alpha}{h_{1} h_{2}}\left((-1)^{k+m} \gamma \sqrt{\alpha /(1-\nu)}+\sqrt{-8 \gamma h_{2}^{3}}\right) .
$$

Therefore we conclude that this determinant is zero if and only if either $\alpha \gamma=0$, or $k+m$ is odd and $\alpha \gamma=-8(1-\nu) h_{2}^{3}$. So, Theorem 2 is proved.

6.3. $S_{23}$-symmetric periodic solutions. By Proposition 4 the solution $\varphi\left(s ; \xi_{10}, \xi_{20}, \eta_{10}, \eta_{20},(\alpha, \beta, \gamma, \nu, \mu)\right)$ of system (4) for $\mu=0$ is a $S_{23}$-symmetric if the initial conditions satisfy

(a) either $\xi_{1}(0)=\xi_{10}^{*}, \xi_{2}(0)=0, \eta_{1}(0)=0, \eta_{2}(0)=\eta_{20}^{*}$;

(b) or $\xi_{1}(0)=\xi_{10}^{*}, \xi_{2}(0)=\xi_{20}^{*}, \eta_{1}(0)=0, \eta_{2}(0)=0$;

for fixed values of $\alpha, \beta, \gamma, \nu$ and $h$.

6.3.1. Case (a). By the statement (c) of Proposition 1 we have that the solution $\varphi$ is a $S_{23}$-symmetric periodic solution of the charged collinear 3body problem with period $S$ satisfying the energy condition $G=0$ if and only if

$$
\begin{array}{ll}
\xi_{1}\left(S / 4 ; \xi_{10}, \eta_{20},(\alpha, \beta, \gamma, \mu)\right) & =0, \\
\eta_{2}\left(S / 4 ; \xi_{10}, \eta_{20},(\alpha, \beta, \gamma, \mu)\right) & =0, \\
G\left(\xi_{20}, \eta_{10},(\alpha, \beta, \gamma, \mu)\right) & =0 .
\end{array}
$$

Solving the last equation in terms of $\eta_{10}$ we obtain

$$
\eta_{20}=\sqrt{\frac{8 \gamma \nu(1-\mu)}{1-\mu+\mu \nu}}
$$

So the solution $\varphi\left(s ; \xi_{10}, 0,0, \eta_{20},(\alpha, \beta, \gamma, \nu, \mu)\right)$ is a $S_{23}$-symmetric periodic solution of system (4) satisfying $G=0$ if and only if

$$
\begin{aligned}
& \xi_{1}\left(S / 4 ; \xi_{10},(\alpha, \beta, \gamma, \mu)\right)=0 \\
& \eta_{2}\left(S / 4 ; \xi_{10},(\alpha, \beta, \gamma, \mu)\right)=0 .
\end{aligned}
$$


Statement $(c)$ of Proposition 4 provides the initial conditions and the numbers $p$ and $q$, see Table 1 in order to the periodic orbits be symmetric. More precisely, if $p=2 m, q=2 k+1, S=S^{*}=s\left(p T_{1}\left(h_{1}, \alpha\right)\right)=s\left(q T_{2}\left(h_{2}, \gamma\right)\right)$, $\xi_{10}^{*}=\sqrt{\frac{\alpha}{-h_{1}}}$ and $\eta_{20}^{*}=\sqrt{8 \gamma \nu}$, with $m$ and $k$ positive integers, then $\varphi\left(s ; \xi_{10}^{*}, 0,0, \eta_{20}^{*},(\alpha, \beta, \gamma, \nu, 0)\right)$ is a $S_{23}$-symmetric solution for system (4) for $\mu=0$. Moreover the energy level $H=h=h_{1}+h_{2}$ satisfies the condition $h_{1}=\left(\frac{p \alpha}{q \gamma}\right)^{\frac{2}{3}} h_{2}\left(\frac{1-\nu}{\nu}\right)^{\frac{1}{3}}$ given in statement $(c)$ of Proposition 3. Our objective now is extend this solution for $\mu>0$ and small. Applying the Implicit Function Theorem in system (24) in a neighbourhood of a known solution we have that if

$$
\left|\begin{array}{ll}
\frac{\partial \xi_{1}}{\partial s} & \frac{\partial \xi_{1}}{\partial \xi_{10}} \\
\frac{\partial \eta_{2}}{\partial s} & \frac{\partial \eta_{2}}{\partial \xi_{10}}
\end{array}\right| \begin{array}{ll}
\mid \begin{array}{ll}
s & =S^{*} / 4 \\
\xi_{10} & =\xi_{10}^{*} \\
\mu & =0
\end{array}
\end{array} \neq 0
$$

then there exist unique analytic functions $\eta_{10}=\eta_{10}(\mu)$ and $S=S(\mu)$ defined for $\mu \geq 0$ sufficiently small that satisfy

(i) $\eta_{10}(0)=0$ and $S(0)=S^{*}$,

(ii) and $\varphi\left(s ; \xi_{10}, 0,0, \eta_{20}, \mu\right)$ is a $S_{23}$-symmetric periodic solution of system (4) with period $S=S(\mu)$ satisfying the energy condition $G=0$.

We have

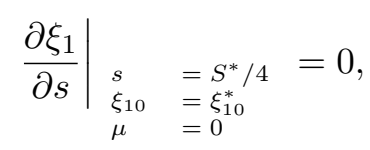

and

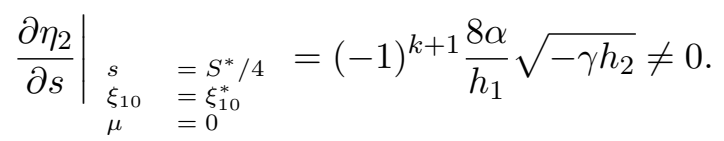

So we have to calculate the derivative $\partial \xi_{1} / \partial \xi_{10}$ evaluated at $s=S^{*} / 4, \xi_{10}=$ $\xi_{10}^{*}$ and $\mu=0$. As in the previous cases we obtain that

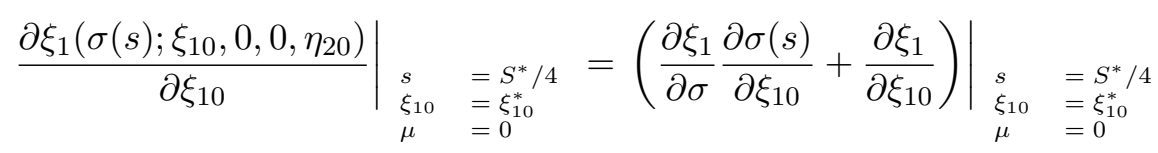

is $(-1)^{m+1}$. Now determinant $(23)$ is

$$
(-1)^{m+k} \frac{8 \alpha}{h_{1}} \sqrt{-\gamma h_{2}} .
$$

Therefore we conclude that the determinant is zero if and only if $\alpha \gamma=0$. 
6.3.2. Case (b). From the second part of statement $(c)$ of Proposition 1 the solution $\varphi$ is a $S_{23}$-symmetric periodic solution with period $S$ satisfying the energy condition $G=0$ if and only if

$$
\begin{array}{ll}
\xi_{1}\left(S / 4 ; \xi_{10}, \xi_{20},(\alpha, \beta, \gamma, \mu)\right) & =0, \\
\xi_{2}\left(S / 4 ; \xi_{10}, \xi_{20},(\alpha, \beta, \gamma, \mu)\right) & =0, \\
G\left(\xi_{10}, \xi_{20},(\alpha, \beta, \gamma, \mu)\right) & =0 .
\end{array}
$$

The solution of the equation $G=0$ in terms of $\xi_{20}$ is given by

$$
\xi_{20}=\sqrt{\frac{\alpha h_{2}-h_{1}(\gamma+\beta \mu)+\sqrt{\left(\alpha h_{2}\right)^{2}+2 h_{1} h_{2} \alpha(\gamma-\beta \mu)+h_{1}^{2}(\gamma+\beta \mu)^{2}}}{2 h_{1} h_{2}}} .
$$

So the solution $\varphi\left(s ; \xi_{10}, \xi_{20}, 0,0,(\alpha, \beta, \gamma, \nu, \mu)\right)$ is a $S_{23}$-symmetric periodic solution of system (4) that satisfies $G=0$ if and only if

$$
\begin{aligned}
& \xi_{1}\left(S / 4 ; \xi_{10},(\alpha, \beta, \gamma, \mu)\right)=0, \\
& \xi_{2}\left(S / 4 ; \xi_{10},(\alpha, \beta, \gamma, \mu)\right)=0 .
\end{aligned}
$$

Again by statement $(c)$ of Proposition 4 we have the initial conditions and the numbers $p$ and $q$ (see Table 1) to have symmetric periodic solutions. That is, $p=2 m, q=2 k+1, S=S^{*}=s\left(p T_{1}\left(h_{1}, \alpha\right)\right)=s\left(q T_{2}\left(h_{2}, \gamma\right)\right), \xi_{10}^{*}=$ $\sqrt{\frac{\alpha}{-h_{1}}}, \xi_{20}^{*}=\sqrt{\frac{\gamma}{-h_{2}}}$, for $\mu=0$. Furthermore the energy level $H=h=$ $h_{1}+h_{2}$ satisfies the condition $h_{1}=\left(\frac{p \alpha}{q \gamma}\right)^{\frac{2}{3}} h_{2}\left(\frac{1-\nu}{\nu}\right)^{\frac{1}{3}}$ given in statement (c) of Proposition 3. As in the previous case applying the Implicit Function Theorem to system (25) in a neighborhood of a known solution we have that if

$$
\left|\begin{array}{ll}
\frac{\partial \xi_{1}}{\partial s} & \frac{\partial \xi_{1}}{\partial \xi_{10}} \\
\frac{\partial \xi_{2}}{\partial s} & \frac{\partial \xi_{2}}{\partial \xi_{10}}
\end{array}\right|_{\mid \begin{array}{ll}
s & =S^{*} / 4 \\
\xi_{10} & =\xi_{10}^{*} \\
\mu & =0
\end{array}} \neq 0
$$

then there exist unique analytic functions $\xi_{10}=\xi_{10}(\mu)$ and $S=S(\mu)$ defined for $\mu \geq 0$ sufficiently small that satisfies

(i) $\xi_{10}(0)=\xi_{10}^{*}$ and $S(0)=S^{*}$,

(ii) and $\varphi\left(s ; \xi_{10}, \xi_{20}, 0,0, \mu\right)$ is a $S_{23}$-symmetric periodic solution of system (4) with period $S=S(\mu)$ satisfying the energy condition $G=0$.

Working as in the previous cases we get

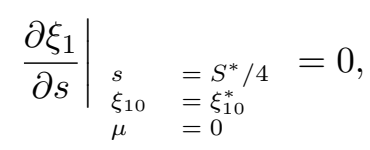


and

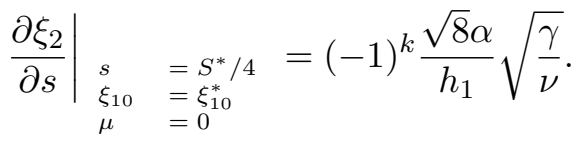

So it remains to calculate $\partial \xi_{1} / \partial \xi_{10}$ evaluated at $s=S^{*} / 4, \xi_{10}=\xi_{10}^{*}$ and $\mu=$ 0 . This derivative is obtained derivating the solution $\xi_{1}\left(\sigma(s) ; \xi_{10}, \xi_{20}, 0,0,0\right)$ of system (5) evaluated at $\xi_{10}=\xi_{10}^{*}$ and $s=S^{*} / 4$ with initial conditions

$$
\xi_{10}=\xi_{10}, \xi_{2}(0)=\xi_{20}, \eta_{1}(0)=0, \eta_{2}(0)=0
$$

satisfying the energy relation $G=0$. Then

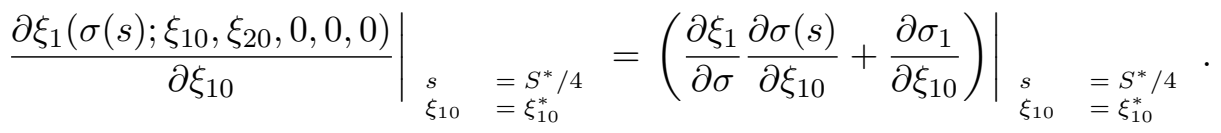

As the previous case, we obtain

$$
\left.\frac{\partial \xi_{1}\left(\sigma(s) ; \xi_{10}, \xi_{20}, 0,0,0\right)}{\partial \xi_{10}}\right|_{\substack{s \\ \xi_{10} \\=S_{10}^{*} / 4}}
$$

is $(-1)^{m}$, and determinant (26) is given by

$$
(-1)^{k+m} \frac{\alpha}{h_{1}} \sqrt{\frac{8 \gamma}{\nu}}
$$

Therefore we conclude that this determinant is zero if and only if $\alpha \gamma=0$. This completes the proof of Theorem 3 .

\section{Concluding Remarks}

In this paper we study the periodic solutions of the collinear charged 3-body problem which are $S_{12^{-}}, S_{13^{-}}$and $S_{23^{-}}$symmetric. Applying the continuation method of Poincaré we obtain that six families of symmetric periodic orbits can be extend from $\mu=0$ to small positive values of $\mu$. In [5] it was studied a similar problem, but in that paper the bodies are uncharged and applying the continuation method of Poincaré only three families of periodic orbits can be extended from $\mu=0$ to $\mu$ small and positive.

If we consider that families the values of the charges $q_{1}, q_{2}, q_{3}$ tends to zero then, for each symmetry $S_{i j}$ considered, we observe that one of the families of periodic orbits converge continuously to one of the families of periodic orbits given in [5], and the other one the continuation method cannot be applied because the determinant of the partial derivatives of the system is zero. In fact, the expression of the charges $q_{i}$ in terms of the parameters 
$\alpha, \beta, \gamma, \mu, \nu$ are given by

$$
\begin{aligned}
& q_{1}(\alpha, \beta, \gamma, \mu, \nu)=\frac{\mu((\mu-1)(\nu-1)-\alpha) \sqrt{\frac{-\beta+(1-\nu) \nu}{\alpha-\mu \nu+\mu+\nu-1}}}{\sqrt{\gamma+(\mu-1) \nu}} \\
& q_{2}(\alpha, \beta, \gamma, \mu, \nu)=\sqrt{\frac{(\alpha-\mu \nu+\mu+\nu-1)(\gamma+(\mu-1) \nu)}{-\beta+(1-\nu) \nu}} \\
& q_{3}(\alpha, \beta, \gamma, \mu, \nu)=-\mu \sqrt{\gamma+(\mu-1) \nu} \sqrt{-\frac{\beta+(\nu-1) \nu}{\alpha-\mu \nu+\mu+\nu-1}} .
\end{aligned}
$$

The charges $q_{1}, q_{2}$ and $q_{3}$ are zero for the value of parameters $\alpha=\alpha^{*}=$ $(\mu-1)(\nu-1), \beta=\beta^{*}=(1-\nu) \nu$ and $\gamma=\gamma^{*}=\nu(1-\mu)$. Considering these values of parameters, we have that determinant (16) is

$$
(-1)^{k+m+1} \frac{8 \pi \nu(2 k+1) \sqrt{-h_{1}(1-\nu)}}{h_{2}}
$$

which is distinct of zero and therefore it is possible to perform the continuation method of Poincaré. With the values of parameters $\alpha^{*}, \beta^{*}$ and $\gamma^{*}$ the initial conditions given in statement $(a)$ of Theorem 1 are $\xi_{2}(0)=\sqrt{-\nu / h_{2}}$ and $\eta_{1}(0)=\sqrt{8}(\nu-1)$ that coincide with the initial conditions of the $S_{12^{-}}$ symmetric periodic solution given in Theorem 5.1 of [5].

However for statement (b) of Theorem 1 the determinant (19) with the parameters $\alpha^{*}, \beta^{*}$ and $\gamma^{*}$ is zero. The same happens for the others periodic symmetric orbits with symmetries $S_{13^{-}}$and $S_{23^{-}}$.

\section{ACKNOWLEDGEMENTS}

The first author is partially supported by a MINECO grant MTM201340998-P, an AGAUR grant number 2014SGR-568, the grants FP7-PEOPLE2012-IRSES 318999 and 316338, and a CAPES grant number 88881.030454/ 2013-01 from the program CSF-PVE. The second author is supported by grant\#2012/10 267000 803, Goiás Research Foundation (FAPEG), PROCAD/CAPES grant 88881.0 68462/2014-01 and by CNPq-Brazil.

\section{REFERENCES}

[1] P. Atela and R. I. McLachlan. Global behavior of the charged isosceles three-body problem. Internat. J. Bifur. Chaos Appl. Sci. Engrg., 4(4):865-884, 1994.

[2] R. Broucke and D. E. Walker. Numerical explorations of the rectilinear problem of three bodies. In Proceedings of the Sixth Conference on Mathematical Methods in Celestial Mechanics (Math. Forschungsinst., Oberwolfach, 1978), Part I, volume 21, pages 73-81, 1980.

[3] J. Casasayas and J. Llibre. Qualitative analysis of the anisotropic Kepler problem. Mem. Amer. Math. Soc., 52(312):viii+115, 1984.

[4] J. Casasayas and A. Nunes. A restricted charged four-body problem. Celestial Mech. Dynam. Astronom., 47(3):245-266, 1989/90. 
[5] M. Corbera and J. Llibre. Developments in Mathematical and Experimental Physics: Volume C: Hydrodynamics and Dynamical Systems, chapter Symmetric Periodic Orbits for the Collinear 3-body Problem Via the Continuation Method, pages 117-141. Springer US, Boston, MA, 2003.

[6] M. Corbera and J. Llibre. Periodic orbits of a collinear restricted three-body problem. Celestial Mech. Dynam. Astronom., 86(2):163-183, 2003.

[7] M. Corbera, J. Llibre, and E. Pérez-Chavela. Symmetric periodic orbits near a heteroclinic loop formed by two singular points and their invariant manifolds of dimension 1 and 2. J. Phys. A, 39(50):15313-15326, 2006.

[8] Donald L. Hitzl and Michel Hénon. Critical generating orbits for second species periodic solutions of the restricted problem. Celestial Mech., 15(4):421-452, 1977.

[9] R. McGehee. Triple collision in the collinear three-body problem. Invent. Math., 27:191-227, 1974.

[10] K. R. Meyer. Periodic solutions of the N-body problem, volume 1719 of Lecture Notes in Mathematics. Springer-Verlag, Berlin, 1999.

[11] K. R. Meyer and G. R. Hall. Introduction to Hamiltonian dynamical systems and the $N$-body problem, volume 90 of Applied Mathematical Sciences. Springer-Verlag, New York, 1992.

[12] E. Perez-Chavela, D. G. Saari, A. Susin, and Z. Yan. Central configurations in the charged three body problem. In Hamiltonian dynamics and celestial mechanics (Seattle, WA, 1995), volume 198 of Contemp. Math., pages 137-153. Amer. Math. Soc., Providence, RI, 1996.

[13] H. Poincaré. Les méthodes nouvelles de la mécanique céleste. Tome III. Invariants intégraux. Solutions périodiques du deuxième genre. Solutions doublement asymptotiques. Dover Publications, Inc., New York, N.Y., 1957.

[14] M. Santoprete. Symmetric periodic solutions of the anisotropic Manev problem. J. Math. Phys., 43(6):3207-3219, 2002.

${ }^{1}$ Departament de Matemàtiques, Universitat Autònoma de Barcelona, 08193 Bellaterra, Barcelona, Spain

E-mail address: jllibre@mat.uab.cat

2 Institute of Mathematics and Statistics of Federal University of Goiás, Avenida Esperança s/n, Campus Samambaia, CEP 74690-900, Goin̂nia, Goiás, BRAZIL.

E-mail address: djtonon@ufg.br 